\title{
La resolución de conflictos: una estrategia de formación hacia la convivencia para la paz
}

\author{
Conflict resolution: a training strategy towards coexistence for peace
}

\author{
Sara Torres-Hernández • Kenia Janeth Mares López
}

Sara Torres-Hernández. Centro Chihuahuense de Estudios de Posgrado, México. Es doctora en Desarrollo Educativo con énfasis en Formación de Profesores, trabaja las líneas de investigación: resolución de conflictos, convivencia, habilidades socioemocionales y contextos vulnerables. Tiene diferentes participaciones en congresos nacionales e internacionales en Estados Unidos, España, Italia y Argentina. Cuenta con la distinción de la Medalla al Mérito Educativo 2016, otorgada por el $\mathrm{H}$. Congreso del Estado de Chihuahua. Correo electrónico: sara.torres.h@cchep. edu.mx. ORCID: https://orcid. org/0000-0002-6752-6993.

Kenia Janeth Mares López. Centro de Desarrollo Integral Infantil, Zona Sur, Chihuahua, México. Es maestra en Desarrollo Educativo por el Centro Chihuahuense de Estudios de Posgrado y tiene experiencia como docente de primaria y preescolar. Correo electrónico: keniax@ hotmail.com. ORCID: https://orcid. org/0000-0002-1130-3555.

\section{Resumen}

Esta propuesta de intervención ofrece ver al conflicto como una oportunidad de aprendizaje para la transformación positiva de la convivencia. Si bien se aprende a convivir diariamente desde casa, para formarse en construir la paz se requiere trabajar desde las aulas; esto se puede lograr al destacar factores positivos que permitan el cambio y aprender a resolver las diferencias entre los involucrados. Este proyecto favorece los ambientes de aprendizaje, a través de promover la sana convivencia por medio de diversas estrategias para la resolución de conflictos de manera positiva y al fomentar habilidades socioemocionales. Se abordó desde el paradigma sociocrítico, con base en la investigación-acción, es decir, involucrándose en lo que se quiere transformar; para el diagnóstico y la evaluación de las estrategias se aplicaron técnicas como la observación, encuesta, entrevista y tests aplicados a las docentes. Se encontró baja autoestima en el alumnado y que ni los estudiantes ni los docentes saben cómo afrontar ni resolver los conflictos. Para atender esta realidad se aplicaron doce actividades orientadas en habilidades sociales; se fomentó la inteligencia intrapersonal y se manejaron también diversas estrategias de resolución del conflicto, las cuales involucraron a docentes, alumnado y jefes de familia de educación primaria. Los resultados fueron positivos, muestran cambios en los involucrados, en su manera de desenvolverse socialmente, actitudes y formas de pensar más positivas, lo que dio como consecuencia la mejora en la sana convivencia al apreciar al conflicto como una oportunidad para aprender de los otros.

Palabras clave: Convivencia pacífica, conflictos escolares, educación para la convivencia, asertividad, educación emocional.

\section{Abstract}

This intervention proposition offers to look at school conflicts as an opportunity for learning to transform coexistence in a positive manner. Even though individuals learn to coexist in their homes, if we wish to create peace, it is required for it to be worked within the classroom; this can be achieved by highlighting positive factors that enable change and learning to resolve the differences between those involved. This project favors learning environments, by promoting healthy coexistence through diverse strategies for positive conflict resolution and promoting social-emotional abilities. This topic 
was approached from a socio-critical paradigm, based on action research, in other words, getting involved in what is desired to transform; for the diagnosis and evaluation of the strategies, techniques such as observation, polls, interviews, and tests applied by teachers were implemented. Low self-esteem within the students was found, and that neither students nor teachers knew how to face or resolve conflicts. To attend this reality, twelve social-oriented skills activities were applied; intrapersonal intelligence was promoted, and diverse conflict resolution strategies were used, in which teachers, students, and elementary education family leaders were involved. The results were positive, the participants demonstrated changes in their way of developing socially, their attitude and more positive ways of thinking, which resulted in an improvement in healthy coexistence by appreciating the conflict as an opportunity to learn from others.

Keywords: Peaceful coexistence, school conflicts, education for coexistence, assertiveness, emotional education.

\section{INTRODUCCIÓN}

La convivencia es importante dentro del ambiente escolar, ya que ayuda a elevar la calidad educativa y se traduce en beneficios sobre la comunidad escolar. Las escuelas son lugares de formación, en los que el docente al promover educación socioemocional en el alumnado puede hacer conciencia sobre la responsabilidad en la toma de decisiones y en la resolución de conflictos. El clima emocional tiene relación con el ambiente positivo, ya que es un factor clave dentro de la convivencia escolar, este se percibe por los miembros de la comunidad educativa, ya que determina los estilos de convivencia, toma en cuenta aquellas relaciones interpersonales, ya sea de colaboración y cooperación, en donde existan acuerdos pertinentes, se vivan valores institucionales coherentes y se promueva la participación libre, esto con el fin de implementar una sana convivencia.

Aprender a convivir implica una serie de habilidades y competencias personales y sociales, así mismo exige la existencia de normas y valores consensuados, un reconocimiento y respeto por lo diferente, así como el compromiso de los involucrados. La importancia de tomar en cuenta las relaciones que se llevan dentro de la convivencia brinda a la comunidad educativa la oportunidad de reflexionar sobre tener una mirada distinta del conflicto, es decir, si se actúa de manera positiva dentro de la institución, esto generará mayores beneficios en los ambientes de aprendizaje, en la comunidad y con los involucrados.

\section{Diagnóstico}

El presente informe corresponde a uno de tres casos en los cuales se trabajó con el proyecto de intervención: el conflicto como una oportunidad. Para el diagnóstico se aplicó el test sobre las modalidades de resolución de conflictos de Thomas y Kilmann 
(2014), un cuestionario sobre la sana convivencia en el entorno escolar y una encuesta a docentes sobre convivencia y conflictos en el ambiente escolar. Los conflictos que más se presentaron fueron las peleas entre el alumnado, agresiones verbales y físicas, producto de los desacuerdos que hay entre ellos, los apodos, las burlas, los chismes y en ocasiones por juegos violentos, así como el robo de materiales o conductas disruptivas. Los docentes mencionan que la convivencia dentro de la institución se vio afectada por las malas relaciones familiares (la mayoría de los alumnos están solos por largas jornadas y la comunicación entre los miembros no es adecuada), porque existe diferencia de valores entre los alumnos, así como por el contexto violento en el que se vive.

Se logró apreciar que dentro de esta institución era necesario trabajar en la sana convivencia, ya que la mayoría de los docentes menciona que la manera en la que se relacionan no era adecuada, porque frecuentemente había conflictos, si bien se había estado trabajando en ello había que poner mayor énfasis para transformarla de la mejor manera posible.

En cuanto a la postura de lo que es el conflicto, la mayoría de las maestras tienen una perspectiva negativa de él, ya que el $89 \%$ opina que el conflicto es un problema que se da entre dos o más personas. Además, la modalidad de resolución de conflictos de la mayoría del profesorado se ubica en el estilo de la evasión, esto quiere decir que no buscan ni sus intereses ni los de la otra persona, prefieren poner a un lado la situación, por lo que se infiere que no se estaba enseñando a afrontar el conflicto.

Se logró observar que las relaciones entre los estudiantes eran intolerantes, por lo que era necesario trabajar sobre la mejor manera de convivir, tomando en cuenta también a las familias, profesores y directivos, ya que al tener una buena relación entre individuos existirá un cambio positivo dentro de la institución. El diagnóstico mostró los problemas de convivencia que se vivían en esta institución; por ello se decide transformar los espacios de aprendizaje en ambientes de sana convivencia dentro de la institución escolar.

Se evidenció la necesidad de fomentar la cooperación, el diálogo, así como el aprecio por las diferencias para tomar decisiones de manera colaborativa y mejorar estos espacios de aprendizaje, ya que si se tiene un clima positivo en el cual exista el diálogo, se tome en cuenta las emociones de los miembros de la institución, se fomenten habilidades, se promueva la empatía, la asertividad, la comunicación de manera respetuosa, beneficia de una manera relevante a las escuelas. Por ello se planteó la pregunta de intervención: ¿Cómo transformar los espacios de aprendizaje en ambientes de sana convivencia?

\section{Objetivo general}

- Generar estrategias de sana convivencia que favorezcan ambientes adecuados para el aprendizaje. 
Preguntas de investigación

- ¿Cómo mejorar los ambientes de aprendizaje?

- ¿Cuáles estrategias permiten desarrollar la sana convivencia?

- ¿Qué habilidades permiten afrontar y resolver los conflictos de manera positiva?

\section{REFERENTES TEÓRICOS}

La convivencia es un proceso que se vive todos los días en el hogar, el área de trabajo, con los amigos y en distintos lugares donde cada persona se relaciona con los demás; este intercambio de experiencias tiene un carácter formativo, el cual se lleva de manera intencional dentro de las escuelas, como una manera de formar desempeños futuros en la vida ciudadana y social (Fierro, Carbajal, y Martínez-Parente, 2010).

La convivencia en general es aquella que, cuando es adecuada, "puede ser conceptualizada como un modo de vivir en relación o en interrelación con otros, en la cual se respeta y considera las características y diferencias individuales de las personas involucradas independientemente de sus roles y funciones" (Benites, Carozzo, Horna, Palomino, Salgado, Uribe y Zapata, 2012, p. 76).

Existen distintas maneras de relacionarse, una de ellas es la sana convivencia; esta es una forma positiva de llevar a cabo relaciones entre individuos, en ella existe la comunicación, se manejan valores y límites, es decir, hay acuerdos, los cuales influyen para que haya respeto mutuo hacia la diversidad; se vive en compañía de otros donde se promueve y existe una unión. Esta interrelación conlleva a situaciones entre las cuales se encuentra el conflicto, este es inevitable ya que cada persona tiene sus propias necesidades, valores, gustos o puntos de vista, pero si se maneja utilizando técnicas y herramientas adecuadas puede transformarse en una oportunidad de cambio hacia algo positivo.

Los conflictos son "situaciones en las que dos o más personas entran en oposición o desacuerdo, porque sus peticiones, valores, intereses, aspiraciones, deseos o necesidades son incompatibles o, al menos, se perciben como tales" (Mas y Torrego, 2014, p. 23); es necesario aprender a relacionarse de una manera distinta para transformarlo de manera positiva y constructiva, observando al conflicto como una oportunidad para aprender.

Esto se relaciona con la acción de educar, ya que los conflictos se presentan en las escuelas, pues las relaciones son más complejas debido a las diferencias sociales, económicas y culturales que vive la sociedad. Kolangui y Parra mencionan que el conflicto "es una forma de conducta competitiva entre personas o grupos. Ocurre cuando dos o más personas compiten sobre objetivos o recursos limitados percibidos como incompatibles" (2013, p. 20).

Si se observa de manera positiva, se puede rescatar que dentro del conflicto hay innovación, se puede aprender algo de las diferencias, esto quiere decir que si hay 
un conflicto también existe una forma de relacionarse de manera armónica. Galtung aboga por una perspectiva positiva, "viéndolo como una fuerza motivadora de cambio personal y social" (en Caballero, 2010, p. 155), tomando en cuenta ciertas habilidades básicas, como la asertividad, empatía, control de la impulsividad y el enojo, así como reconocer las diversas emociones que se presenten para poder afrontar el conflicto. Para llevar a cabo este cambio es necesario identificar los estilos y las estrategias de resolución.

En los estilos de resolución de conflictos se logran observar las diversas maneras de actuar de los individuos; para ello, Thomas y Kilmann (2014) mencionan cinco modalidades basadas en la conducta:

- Competidor: no le gusta cooperar, trabaja de manera asertiva, se sitúa más al poder y le interesa ganar, poniendo como prioridad sus propios intereses.

- Colaborador: es asertivo y cooperativo, el individuo trabaja de manera colaborativa, tratando de buscar una solución que satisfaga plenamente a ambos.

- Compromiso: se lleva acabo entre la asertividad y la cooperación, la persona intenta encontrar una solución en la que ambos estén de acuerdo.

- Eludir: no es asertivo ni cooperativo, el individuo no busca ni sus intereses ni los de la otra persona, prefiere poner a un lado la situación.

- Complaciente: no es asertivo, pero sí cooperativo, deja de lado sus propios intereses para satisfacer los de la otra persona; opinan que los conflictos deben resolverse con ayuda, son personas generosas las cuales ven a sus compañeros como amigos, piensan en ellos ubicando como prioridad a la compasión y la amistad.

Si se busca resolver los conflictos de manera abierta y positiva, es necesaria la intervención de los miembros de la institución para guiar a los involucrados de manera pacífica. A continuación se analizan cuatro estrategias: arbitraje, diálogo, mediación y negociación.

En el arbitraje, un tercero es el encargado de resolver el conflicto y las partes tienen que acatar la solución, ya que es quien tiene la autoridad y la competencia para emitir decisiones. "El arbitraje es un medio de heterocomposición de los intereses en conflicto, porque el árbitro, un tercero, decide la solución” (Sánchez, 2012, p. 2); este puede ser de utilidad cuando las partes no pudieron hacerlo por sí mismas.

El diálogo es un factor clave para resolver un conflicto, este es un proceso de comunicación que tiene como objetivo la construcción de significados comunes entre personas para un entendimiento mutuo. Dialogar no es solo hablar (el diálogo), contiene un sinnúmero de bases las cuales resuelven o afrontan al conflicto, gracias a él se llega a acuerdos entre personas, se comprende las emociones y los sentimientos, la idea que la otra persona quiere dar a entender a través de las palabras y expresiones, lo que provoca que se superen los malentendidos entre personas. 
Con la escucha activa se expresan las necesidades y deseos eficazmente, cuando se atienden y se interesa por las necesidades de los demás; por lo tanto, escuchar activamente es mostrar apertura ante lo que la otra persona trata de comunicar, atendiendo lo no-verbal, es decir: lo que las personas observan, lo que se ve de la cara y el cuerpo de la otra persona, y lo paraverbal, lo cual se refiere a cómo se dice, a la entonación, proyección y resonancia de la voz (Kolangui y Parra, 2013).

La mediación representa una gran aportación en el campo educativo, es un método para resolver conflictos, gracias a la intervención de una tercera persona justa que es aceptada por los involucrados, no tiene el poder de decidir y es ajena al conflicto, lo cual facilita la comunicación y el diálogo (Pérez y Pérez, 2011).

La negociación es un proceso por el cual dos partes llegan a un acuerdo con el que están satisfechas, no necesita de terceras personas. Ambas ceden y ganan algo. El objetivo consiste "para dos o más partes en conflicto, en llegar a un acuerdo por medio de conversaciones e intercambio de opiniones entre sus representantes" (Manzano y Torres, 2000, p. 32). Existe una motivación de llegar a un acuerdo de manera armónica entre los involucrados.

En cuanto a la inteligencia emocional, cada día se descubren más detalles bioquímicos y fisiológicos para explicar cómo es que una emoción prepara al organismo para una clase distinta de respuesta (Esquivel, 2005). Esto indica que conforme las personas adquieran diversas perspectivas se genera un cambio, es decir, si se entiende el conflicto como una oportunidad dentro de la sana convivencia, las personas pueden alcanzar un nivel de paz, utilizando las habilidades básicas que se promueven con base en las distintas estrategias que este trabajo presenta.

De acuerdo a Goleman (2001), la inteligencia emocional se compone de dos: la personal y la interpersonal. La primera cuenta con las siguientes habilidades:

- Autoconocimiento: es el conocimiento de las propias emociones y de la manera en la cual afectan a la persona; es necesario ser consciente de las propias limitaciones y reconocer los errores.

- Autorregulación: es no dejarse llevar por los sentimientos del momento, aprender a reconocer que es pasajero, cuando se desarrolla adecuadamente se regulan las emociones e impulsos, y la persona se hace responsable de sus propios actos, sinceros, íntegros, control de estrés, entre otros.

- Automotivación: es entrar en un estado de continua búsqueda y persistencia, encontrando soluciones pertinentes; son personas que muestran gran entusiasmo por sus actividades laborales o académicas, y tienen un alto grado de iniciativa y compromiso, son optimistas en la consecución de sus objetivos.

Así mismo, la inteligencia interpersonal contempla las siguientes habilidades:

- Relaciones interpersonales: es poder desarrollar y mantener una buena relación con los demás, saber convivir con aquellos que están en una posición diferente. 
- Habilidades sociales: es el manejo de las relaciones con los demás, saber persuadir e influenciar a otros.

- Empatía: capacidad e ponerse en el lugar de otras personas.

Es necesario tomar en cuenta toda aquella información que se relaciona con el conflicto y verlo de manera positiva, entenderlo y desarrollar aquellas habilidades básicas mencionadas anteriormente, para lograr un espacio de sana convivencia, el cual puede favorecer los ambientes adecuados para el aprendizaje de los alumnos, tomando en cuenta también las estrategias para tener mayor comprensión de la importancia de presentar este tipo de proyectos.

\section{RUTA METODOLÓGICA Y RESULTADOS}

En el paradigma sociocrítico es necesario unir la teoría y la práctica para lograr tener una visión global de la situación, ya que tiene la finalidad de transformar las estructuras de las relaciones sociales y dar respuesta a problemas generados de la misma (Sandín, 2003).

Desde el paradigma sociocrítico la finalidad es recopilar información sobre la realidad que permita tomar decisiones para la mejora o transformar la realidad analizada, en esta indagación se hace participar a la comunidad estudiada en la medida que toman conciencia sobre su participación en la transformación. Se establece una relación entre lo deseable y lo posible [Torres-Hernández, 2017, p. 71].

El cambio se promueve con la investigación-acción, es decir, entrando a fondo dentro de lo que se quiere solucionar, encontrar en la institución aquellos factores positivos para partir de ahí, buscar cómo son las relaciones entre los individuos, observarlas, saber qué conocen sobre convivencia y diálogo, e identificar los valores que se fomentan para poder poner en marcha lo que se desea transformar. De acuerdo con Mckernan (en Álvarez, 2003), la investigación-acción se fundamenta de tres pilares indispensables en su desarrollo: la participación de los sujetos, la conducta naturalista y su metodología cualitativa.

La investigación-acción se convirtió, por tanto, en el método adecuado, ya que permitió autorreflexión de los involucrados y la toma de decisión para la participación en los cambios; se partió de un diagnóstico, en el cual se detecta una problemática, se plantea una estrategia de solución, se aplica el plan y se evalúan resultados.

El presente proyecto de intervención buscó mejorar la sana convivencia a través de la resolución de conflictos de manera positiva, observándola como una oportunidad para aprender; está dirigido a estudiantes de educación primaria y a jefes de familia; se aplicaron 12 estrategias basadas en el diálogo, la mediación, el arbitraje y la negociación, así como en habilidades básicas como la empatía, la asertividad y la inteligencia emocional; es un proyecto dinámico y motivador para los involucrados ya que cada una de las estrategias tienen un tiempo aproximado de una hora y media. 
El contexto en el que se llevó a cabo es de violencia, con condiciones de marginalidad social, cultural y económica; la diversidad de tipos de familia y su acercamiento a la escuela dificultan no solo los procesos de comunicación sino los de aprendizaje y desarrollo de los estudiantes; la escuela está en una colonia aledaña al Centro de Rehabilitación Social (CERESO) de la ciudad de Chihuahua.

La primera actividad, Tomemos acuerdos, estuvo dirigida hacia la toma de compromisos de convivencia; ser parte de las normas que regulan la participación ayudó a que se introyectaran y fueran motivo de autorregulación. Ya que se establecieron, se trabajó en que el alumnado expresara el conocimiento de las propias emociones y la manera en que les afectan. Para ello fue necesario que se conociera cuáles son, sus funciones y cómo podían regularlas. Se reconocieron cualidades propias y de otros para generar empatía, esta fue una de las actividades que mayor impacto tuvo en el autoconcepto del alumnado, pues les permitió reconocerse y saber que otras personas ven también cualidades en ellos o ellas.

La actividad denominada Busquemos el tesoro se enfocó en desarrollar y fortalecer las habilidades sociales y relaciones interpersonales, lo que ayudó a mejorar la comunicación, la convivencia entre los estudiantes y la comprensión hacia los demás; favoreció además la autorregulación y el fomento de la tolerancia; se trabajó la respiración como proceso para relajarse.

Una Caja de abrazos fue una actividad permanente, en la que diariamente se escogía un tipo de abrazo en el grupo y se compartía por todos, para desarrollar y fortalecer las habilidades sociales y relaciones interpersonales, confianza y comunicación entre los alumnos, también compartir sentimientos de afecto y expresión emocional sin palabras; esta actividad era de las más esperadas por los niños y niñas, con ella se estrecharon lazos de amistad; fue un gran logro, pues al inicio del proyecto no había muchas muestras de afecto entre ellos. Los reporteros es una actividad de juego de roles que ayudó a conocer las diferentes estrategias de resolución de conflictos, pues tuvieron la responsabilidad de decidir cuál era la manera más adecuada y así estar todos conformes con el resultado; al principio buscaban la intervención del docente, pero al final pudieron dialogar y negociar.

La muralla y el puente fueron retos de comunicación, ya que para pasarlos era necesario emplearla de manera asertiva; estas actividades implicaron un desafío emocional, pero fue satisfactorio observar la deleite al cumplir el reto. Con el juego de Las sillas al revés el objetivo es aprender a trabajar en colaboración, al ponerse de acuerdo en las maneras de resolver un conflicto grupal; el resultado da cuenta de la creatividad y simpleza con la que se podían solucionar situaciones cuando se trataba de que todos ganaran.

Con los responsables de familia se trabajó El recipiente de agua, para fomentar la comunicación al momento de afrontar un conflicto; la participación de los asistentes fue entusiasta, les ayudó a ser empáticos con sus hijos o hijas. Viviendo el presente, cuyo objetivo era concientizar sobre la importancia de la inteligencia emocional, les permi- 
tió ser conscientes del afecto que les brindan y de cómo esto genera mayor impacto a la hora de desenvolverse con las demás personas; los resultados fueron positivos, pues se solicitaron más talleres de este tipo, se resaltó la importancia de trabajar en colaboración familia y escuela.

El objetivo de Carta a mi hijo es concientizar a los jefes de familia sobre la importancia de la relación que tienen con sus hijos, así como el afecto que les brindan. Aunque no todos asistieron, fue un momento emotivo, ya que la carta fue entregada al alumnado en un cierre de actividades junto con los abrazos.

\section{CONCLUSIONES}

Al concientizar sobre la importancia de las emociones se reconoció a la alegría como la más aceptada, debido a que la gente se siente más cómoda con alguien que la expresa, sin embargo, otras como la ternura se les dificultaron; aquí se observa la influencia del contexto, ya que, al ser un entorno duro, con nivel socioeconómico difícil y violento, el alumnado desarrolla actitudes defensivas y poco empáticas.

Las emociones son parte del ser humano, por ello fue importante concientizar que cada una de ellas es indispensable para responder a diversas situaciones dentro del ámbito en el que se desenvuelven; en particular en la escuela, si se logra un equilibrio en las emociones de las personas se generan mejores ambientes de aprendizaje.

$\mathrm{Al}$ fortalecer las relaciones entre el alumnado se manifestó un estado de alegría, el cual benefició el entorno, mejorando las relaciones y actitudes, logrando que se mejoraran los ambientes de aprendizaje.

Es importante que se involucre a jefes de familia, maestros y directivos en los procesos de formación y reconocimientos de la inteligencia emocional, ya que esto provoca que se den mejores resultados al mostrar motivación y entusiasmo para trabajarla no solo en la escuela sino en la casa con sus hijos.

$\mathrm{Al}$ fomentar la autorregulación emocional, los alumnos lograron comprender cómo tener un equilibrio con sus emociones los llevó a reforzar las relaciones entre compañeros, mejorando la sana convivencia entre ellos.

Al compartir los sentimientos de afecto se consiguió fomentar en los estudiantes el autoconocimiento, ya que esto hace que se reflexione acerca de cómo son, lo que los afecta y lo que los hace felices; esto provocó que se conozcan entre todos los involucrados, destacando las habilidades y cualidades positivas, estimulando una sana convivencia dentro del ámbito escolar.

Cuando el alumnado es capaz de reconocer las cualidades de sus compañeros, se consigue identificar la importancia de la empatía, reflexionando cómo al tomar en cuenta a los demás, ponerse en su lugar y escucharlos, se puede llegar a una solución y con esto afrontar los conflictos de manera constructiva.

Involucrar la inteligencia emocional y ver al conflicto como una oportunidad ayudó a mejorar la convivencia no solo escolar sino familiar, ya que se reconoce que 
los conflictos surgen o pueden surgir por una emoción mal manejada. Los estudiantes, al saber cómo regular sus emociones y ponerse metas, aprendieron a vivir de manera más pacífica.

La metodología que se emplea en este proyecto para la resolución del conflicto y de educación socioemocional parte del principio de reconocer al ser, validar sus emociones y darle la oportunidad de regularlas, y de decidir la participación en esta construcción; lo que realmente impacta en este desarrollo es el dejar ser, permitir las equivocaciones y vivir la experiencia, porque al final es de las que más se aprende.

\section{REFERENCIAS}

Álvarez, J. (2003). Cómo hacer investigación cualitativa. Fundamentos y metodología. México.

Benites, L., Carozzo, J., Horna, V., Palomino, L., Salgado, C., Uribe, C., y Zapata, L. (2012). Bullying y convivencia en la escuela. Lima, Perú.

Caballero, J. (2010). Convivencia escolar. Un estudio sobre buenas prácticas. Revista Pazy Conflictos, 154-169.

Esquivel, L. (2005). El libro de las emociones, son de la razón sin corazón. Barcelona: Debolsillo.

Fierro, C., Carbajal, P., y Martínez-Parente, R. (2010). Ojos que sí ven. Casos para reflexionar sobre la convivencia en la escuela. México: Colección Somos Maestr@s, RLC.

Goleman, D. (2001). La inteligencia emocional. Por qué es más importante que el cociente intelectual. México: Ediciones B.

Kolangui, N. T., y Parra, R. L. (2013). El diálogo para la resolución de conflictos. Por una educación para la pa: Guia de actividades para el docente. México: Limusa.

Manzano, M., y Torres, C. (2000). La negociación, una alternativa en la solución de conflictos. Santa Fe.

Mas Ruiz, C., y Torrego Seijo, J. C. (2014). Asesoramiento y mediación de conflictos en los procesos de mejora escolar. Innovación Educativa, 19-34.

Pérez, G., y Pérez, V. (2011). Aprender a convivir, el conflicto como oportunidad de crecimiento. Madrid: Narcea/De Ediciones.

Sánchez, J. (2012). El arbitraje y la mediación como medio de solución de conflictos. Madrid.

Sandín, E. M. (2003). Investigación cualitativa en educación. Fundamentos y tradiciones. Madrid: McGraw-Hill Interamericana.

Thomas, K., y Kilmann, R. (2014). Instrumento de modos de conflicto de Thomas-Kilmann. E.U: Copyright.

Torres-Hernández, S. (2017). Paradigmas en la investigación social. Staobil lekilal ta lekil abtel. Administración para el Desarrollo, (13), 57-76. Recuperado de: http://revistaadministracionfcaunach.mx/archivos/revista_1/numero_13/5.ARTICULO_3_PARADIGMAS_INV_SOCIAL.pdf.

Cómo citar este artículo:

Torres-Hernández, S., y Mares López, K. J. (2020). La resolución de conflictos: una estrategia de formación hacia la convivencia para la paz. Revista Electrónica Científica de Investigación Educativa, 5(1), pp. 119-128. doi: doi.org/10.33010/recie.v5i1.1044. 